\title{
EVALUACIÓN DE LA CALIDAD DE LA CIRUGÍA LAPAROSCÓPICA VIDEO-ASISTIDA PARA EL TRATAMIENTO DEL CÁNCER DE COLON
}

\author{
ASSESSMENT OF THE QUALITY OF VIDEO-ASSISTED LAPAROSCOPIC \\ SURGERY FOR TREATMENT OF COLON CANCER
}

Ameth G. Alvarez-Flores ${ }^{1, a, b}$

\begin{abstract}
RESUMEN
Progresivamente el tratamiento laparoscópico para el cáncer de colon se realiza en más instituciones y en mayor número de pacientes. La definición de estándares de calidad publicados recientemente para este procedimiento permite realizar una autoevaluación y mejora continua. Objetivos: El objetivo del estudio es evaluar el cumplimiento de los estándares propuestos. Métodos: Se realizó un estudio descriptivo y retrospectivo, de pacientes que fueron operados de Cancer de Colon por Laparoscopia Video-asistida, durante el año 2016. Quedaron excluidos: cirugías abiertas, conversiones y procedimientos paliativos. Se evaluaron 4 indicadores principales y otros indicadores secundarios intraoperatorios y postoperatorios, para evidenciar si se encontraban en el valor estándar propuesto. Resultados: Fueron incluidos 12 pacientes. Indicadores de calidad principales: mediana de ganglios: 21, la mediana de estancia hospitalaria postoperatoria: 5.5 días, Mortalidad postoperatoria y casos con fuga de anastomosis ausentes. Conclusión: En el grupo de estudio evaluado se cumplieron los estándares de calidad propuestos.
\end{abstract}

Palabras clave: Calidad; Cirugía; Laparoscópica; Cáncer; Colon. (fuente: DeCS BIREME)

\begin{abstract}
Progressively the laparoscopic treatment for colon cancer is performed in more institutions and in a greater number of patients. The definition of recently published quality standards for this procedure allows for selfassessment and continuous improvement. Objective: The objective of this study is to evaluate compliance with the proposed standards. Methods: A descriptive and retrospective study was performed of patients who underwent colon cancer by video-assisted laparoscopy during the year 2016. The following were excluded: open surgeries, conversions and palliative procedures. Four main indicators and other intraoperative and postoperative secondary indicators were evaluated to show if they were in the proposed standard value. Results: Twelve patients were included. Main quality indicators: median nodes: 21, median postoperative hospital stay: 5.5 days, postoperative mortality and cases with missing anastomosis. Conclusions: In the study group evaluated, the proposed quality standards were met.
\end{abstract}

Key words: Quality; Surgery; Laparoscopic; Cancer; Colon. (source: MeSH NLM)

\footnotetext{
'Hospital Nacional Policía Nacional del Perú “Luis Nicasio Sáenz".

a Médico cirujano.

b Especialista en Cirugía General.

Correspondencia: Ameth G. Alvarez Flores. Correo: amethcirugia@hotmail.com
}

Citar como: Ameth G. Alvarez-Flores. Evaluación de la calidad de la cirugía laparoscópica video-asistida para el tratamiento del cáncer de colon. [Artículo Original]. Rev. Fac. Med. Hum. 2017;17(1):15-18. DOl10.25176/RFMH.v17.n1.743 


\section{INTRODUCCIÓN}

La frecuencia de cáncer de colon y recto es variable según el país; en USA es el tercero tanto en varones como en mujeres ${ }^{1} y$ en Japón es el tercero en hombres y el segundo en mujeres ${ }^{2}$.

La base de la terapia es la resección quirúrgica del tumor ${ }^{3}$. En 1991 el Dr. Moisés Jacobs publico la primera serie de casos en los que se realizó Colectomía Laparoscópica para patología benigna y maligna, que incluyo 12 pacientes con diagnóstico de adenocarcinoma o adenoma de colon. Obtuvo como resultados menor dolor postoperatorio, un retorno rápido de la función intestinal, hospitalización más corta, regreso a la actividad normal más rápido, recuperación postoperatoria más rápida y mejor resultado cosmético. Demostrando que este procedimiento es factible y augurando que sería en el futuro tan común como la colecistectomía laparoscópica 4 .

El 2004 Nelson y colaboradores publicaron los resultados del estudio que comparaba la cirugía abierta versus la cirugía laparoscópica para el tratamiento del cancer de colon, evidenciando que la cirugía laparoscópica obtiene los mismos resultados que la Cirugía Abierta a largo plazo (media de seguimiento 4.4 años) en sobrevida global, tiempo libre de enfermedad, aparición de recidivas, incluida la recidiva en los puntos de incisión quirúrgica concluyendo que la seguridad de ambas técnicas es similar ${ }^{5}$.

El 2005 en Lancet Oncology, Veldkamp y col. publicaron los resultados de un estudio multicéntrico que comparo los resultados a corto plazo (28 días) entre cirugía abierta versus cirugía laparoscópica para el tratamiento del cáncer de colon, no encontrando diferencia entre ambos grupos para la resección ganglionar y bordes de sección seguros. La colectomía laparoscópica se asoció con recuperación temprana, menos analgésicos y menor estancia hospitalaria. Mortalidad y morbilidad similar. Concluyendo que el abordaje laparoscópico es seguro y cumple criterios de radicalidad ${ }^{6}$.

El 2016 Laudicella y col, publicaron un estudio donde se calculó la diferencia en los costos hospitalarios entre la cirugía laparoscópica y la cirugía abierta para el cáncer de colon. Encontraron menor estancia hospitalaria y menos casos de reingreso en el grupo operado por laparoscopia, lo cual produjo un ahorro de £29.3 millones para el Servicio de Salud Ingles ${ }^{7}$.

Luego de haberse establecido que la cirugía Laparoscópica para cáncer de colon es factible, segura, con sobrevida adecuada y más económica en comparación con la cirugía abierta, faltaba una herramienta para que dentro de nuestros servicios quirúrgicos evaluemos la calidad de nuestra cirugía. El Dr. Jorge Sancho ${ }^{8}$ y el ANACO Study Group presentaron en Enero de 2017 un trabajo donde postulan parámetros para evaluar los estándares de calidad de la Cirugía Oncológica de Colon. Los 4 principales indicadores de calidad que propuestos son: mediana de fuga anastomótica menor de $8.5 \%$, Mortalidad menor de $2.5 \%$, mediana de ganglios aislados mayor de 15,1 y mediana de estancia hospitalaria postoperatoria menor de 7,7 días. Presentan otros indicadores de calidad secundarios (intraoperatorios y postoperatorios).

Nuestro Objetivo es Evaluar la Calidad del tratamiento Laparoscópico Video-asistido para Cáncer Colon en el Departamento de Cirugía General del Hospital Nacional PNP "Luis Nicasio Sáenz" durante el 2016 y describir las características clínicas y los resultados oncológicos a corto plazo de este grupo de pacientes.

\section{MÉTODOS}

Estudio Descriptivo y Retrospectivo, con un diseño tipo serie de casos.

Se incluyeron los pacientes operados por Cancer de Colon, durante el periodo 01 de enero al 31 diciembre del 2016.

Criterios de Exclusión fueron: Historia sin datos completos, Colectomía Abierta y/o Conversión, Realización de procedimiento paliativo, Presencia de Cáncer de Recto.

Para la recolección de datos se empleó una ficha elaborada en función a los indicadores de Calidad propuesta por el ANACO study Group, donde se incluyeron variables de:

Características Clínicas: Edad, genero, ASA, antecedente de cirugía previa, localización del tumor, Estadio Clínico y valor del Antígeno Carcino Embrionario (CEA).

Indicadores de Calidad Principales: Fuga anastomótica, Mortalidad, Número de ganglios aislados, Número de días de estancia hospitalaria postoperatoria.

Indicadores de calidad secundarios Intraoperatorios: Transfusión perioperatoria, Complicación Intraoperatoria.

Indicadores de calidad secundarios Postoperatorios y Patológicos: Complicación postoperatoria, infección de herida, Re-intervención, mortalidad por fuga de anastomosis, Resección Ro.

La mortalidad fue definida como muerte dentro de los 60 días del post operatorio. 
Luego de verificar el correcto llenado de las fichas, se elaboró una Base de Datos en M - Excel. Los resultados se presentaron en tablas. Para las variables continuas se emplearon medianas. Para las variables cualitativas se utilizaron porcentajes.

\section{RESULTADOS}

Durante el año 2016 se operaron 12 pacientes de cáncer de colon por cirugía laparoscópica.

Los resultados de las características clínicas se encuentran en la Tabla1 y se describen a continuación: la mediana de edad fue 67 años con rango de $36-81$ años. En cuento al género, predomina en hombres (66\%).La clasificación de ASA muestra la siguiente distribución: ASA | 33 \%, ASA || 50\% y ASA III 16.6\%. 11 pacientes no presentaron antecedente de cirugía previa (91.6\%) siendo la mayoría. El lugar más frecuente de presentación fue el colon derecho (83.3\%) seguido del colon izquierdo (16.6\%). La presentación del Estadio Clínico fue: EC I 50 \%, EC II 25\% y EC III 25. El valor del ACE en el $66 \%$ de pacientes fue mayor o igual a $5 \mathrm{ng} / \mathrm{ml}$ y en el $33 \%$ menor a $5 \mathrm{ng} / \mathrm{ml}^{9}$.

Tabla 1. Características clínicas de los pacientes operados de cáncer de colon.

\begin{tabular}{lc} 
Edad (años) & \\
\hline Mediana (rangos) & $67(36-81)$ \\
\hline Sexo (\%) & \\
\hline Masculino & $66 \%$ \\
Femenino & $33 \%$ \\
ASA(\%) & \\
I & $33 \%$ \\
II & $50 \%$ \\
III & $16.60 \%$ \\
Cirugia Abdminal Previa & \\
No & $11(91.6 \%)$ \\
Una & $1(8.4 \%)$ \\
Localización del Tumor & \\
Colon Derecho & $10(83.3 \%)$ \\
Colon Izquierdo & $2(16.6 \%)$ \\
\hline Estadiaje TNM (\%) & \\
I & $50 \%$ \\
II & $25 \%$ \\
III & $25 \%$ \\
CEA Preoperatorio(ng/ml) & \\
\hline 55 & $66 \%$ \\
\hline 5 & \\
\hline
\end{tabular}

Se realizaron: Hemicolectomia Derecha en 8 pacientes, Hemicolectomia Izquierda en 1 paciente, Sigmoidectomía en 2 pacientes y Transversectomia en 1 paciente. La mediana de Tiempo operatorio fue 300 minutos con un rango de 240 - 420 minutos. La mediana de pérdida sanguínea fue $200 \mathrm{ml}$.

Los valores de los indicadores de calidad principales se muestran en la tabla 2, a continuación. La mediana de ganglios disecados fue 21 , con un rango de 12 - 32 ganglios. La mediana de estancia Hospitalaria post operatoria fue de 5.5 días, con rango de 5 - 23 días. No se presentaron casos de mortalidad y fuga de anastomosis. La mediana de seguimiento fue de 6.5 meses.

Con respecto a los indicadores secundarios intraoperatorios, un caso presento sangrado intraoperatorio que requirió transfusión sanguínea, representando el $8.5 \%$.

Los indicadores secundarios post- operatorios y patológicos se presentaron de la siguiente forma: Infección de herida quirúrgica $8.3 \%$, complicaciones postoperatorias 25\%, re-intervención 8.3\%, Resección Ro en el $100 \%$ y mortalidad $0 \%$.

Tabla 2. Indicadores de calidad principales.

\begin{tabular}{|lc|}
\hline Indicador & $\begin{array}{c}\text { Resultados } \\
\text { (mediana) }\end{array}$ \\
\hline Principales & 21 \\
\hline Número de ganglios & 0 \\
\hline \% Fuga de Anastomosis & 5.5 \\
\hline $\begin{array}{l}\text { Estancia Hospitalaria } \\
\text { Post Operatoria (días) }\end{array}$ & 0 \\
\hline Mortalidad Post Operatoria & \\
\hline
\end{tabular}

\section{DISCUSIÓN}

En los últimos años la preocupación de los centros que realizan colectomía laparoscópica para cáncer de colon está centrada en la calidad de la cirugía que estos pueden ofrecer. Los indicadores de calidad y rangos que propone el ANACO Study Group de España parecen ser una iniciativa útil para poder autoevaluarnos y mejorar de forma continua.

Con respecto a los indicadores principales podemos decir que nuestros resultados se encuentran dentro de los rangos esperados para estos 4 indicadores.

Como se refleja en la tabla 3, el indicador complicación intraoperatoria y transfusión perioperatoria están por 
encima del valor de la mediana. El otro indicador que se presento sobre la mediana fue el porcentaje de re-intervención, presente en el mismo paciente con sangrado intraoperatorio y necesidad de transfusión. Los otros indicadores secundarios se presentaron en valores adecuados respecto a la mediana.

Tabla 3. Comparación de valores de indicadores de calidad secundarios.

\begin{tabular}{|lcl|}
\hline Indicador & $\begin{array}{c}\text { Valores Estándar } \\
\text { (mediana, p25-75) }\end{array}$ & Resultados (mediana) \\
\hline Intraoperatorios & \\
\hline
\end{tabular}

Complicaciones intraoperatorias

\% Transfusión Perioperatoria

Post Operatorias y Patológicos

Infección de Herida Quirúrgica

Complicaciones Post Operatorias

Reintervención

Mortalidad por fuga de Anastomosis

Resección RO

$$
3.4 ; 1.6-6.7
$$

$6.6 ; 2.7-11.4$

8.3

El grupo de pacientes incluidos en este estudio es una población pequeña, que en el corto plazo aumentara y dará mayor consistencia a nuestros resultados.

\section{CONCLUSIÓN}

- En el Departamento de Cirugía General del H.N. PNP Luis N. Sáenz se realiza Cirugía Laparoscópica Video-asistida para el Tratamiento del Cancer de Colon cumpliendo los indicadores de calidad principales propuestos.

- El número de la muestra es una limitante que puede ser resuelta continuando la realización del presente trabajo en el tiempo.

- Continuar con la Cirugía Laparoscópica Videoasistida para el Tratamiento del Cancer de Colon.
- Continuar con el seguimiento de los pacientes incluidos en este estudio para la evaluación de sobrevida, recurrencia y tiempo libre de enfermedad.

Financiamiento: En su totalidad cubiertas por el autor.

Conflicto de interés: El autor manifiesta no tener conflicto de interés alguno en la realización de este estudio.

Recibido: 20 de marzo del 2017

Aprobado: 31 de marzo del 2017

\section{REFERENCIAS BIBLIOGRÁFICAS}

1. Jemal et al; National Cancer Institute's Surveillance, Epidemiology, and End Results (SEER), Bureau of the Census, USA, 2004.

2. Matsuda A and The Japan Cancer Surveillance Research Group. Cancer Incidence and Incidence Rates in Japan in 2007: A Study of 21 Populationbased Cancer Registries for the Monitoring of Cancer Incidence in Japan (MCIJ) Project. Jpn J Clin Oncol; 2013; 43(3):328-336.

3. Vicente J, Solanab A, Alós R. Tratamiento quirúrgico y resultados del cáncer de colon. Cir Esp 2003; 73:20-24.

4. Jacobs M, Verdeja JC, Goldstein HS. Minimally invasive colon resection (laparoscopic colectomy). Surg Laparosc Endosc. 1991 Sep; 1(3):144-50.

5. Nelson $\mathrm{H}$, et al. A Comparison of Laparoscopically Assisted and Open
Colectomy for Colon Cancer. New England Journal Medicine, May 13, 2004; 350(20):2050 -2059.

6. Veldkamp R, Kuhry E, Hop WCJ, Jeekel J, Kazemier G, Bonjer HJ, Haglind E, Pahlman L, Cuesta MA, Msika S, Morino M, Lacy AM. Laparoscopic surgery versus open surgery for colon cancer: short-term outcomes of a randomised trial. Lancet Oncol. 2005 Jul; 6(7): 477 - 84.

7. Laudicella M, et al. BMJ Open 2016;6:1 -8.

8. Sancho J, et al. Resultados quirúrgicos estándar tras resección oncológica de colon. Creación de un nomograma para la autoevaluación. Cirugía Española., January, 2017; 95(1): 30-37.

9. Unidad de Estadística del Hospital Nacional PNP Luis N. Saenz.2015. 